\title{
Pedicle Morphology Using CT-based Navigation System in Adolescent Idiopathic Scoliosis
}

\author{
Shuugo Kuraishi, MD, Jun Takahashi, MD, \\ Hiroki Hirabayashi, MD, Hiroyuki Hashidate, MD, \\ Nobuhide Ogihara, MD, Keijiro Mukaiyama, MD, \\ Hiroyuki Kato, MD
}

Departments of Orthopaedic Surgery,

Shinshu University, School of Medicine,

3-1-1 Asahi, Matsumoto-city, Nagano, 390-8621, Japan

Te1: $81-263-37-2659$

Fax : $81-263-35-8844$

E-mail:kura@apricot.ocn.ne.jp 
Correspondence and requests for reprints to:

Jun Takahashi, M.D.

Departments of Orthopaedic Surgery,

Shinshu University School of Medicine

3-1-1 Asahi, Matsumoto-City, Nagano, 390-8621, Japan

Te1: $81-263-37-2659$

Fax: $81-263-35-8844$

E-mail:jtaka@shinshu-u.ac.jp 


\section{Conflict of Interest Disclosure}

First author: Shuugo Kuraishi, MD - none

Co-authors: Jun Takahashi, MD - none

Hiroki Hirabayashi, MD - none

Hiroyuki Hashidate, MD - none

Nobuhide Ogihara, MD - none

Keijiro Mukaiyama, MD - none

Hiroyuki Kato, MD - none 
Pedicle Morphology in Idiopathic Scoliosis- 1 -

\section{Manuscript text}

\section{Introduction}

Pedicle screw fixation, first described by Boos and Webb [1] in 1959 and popularized by Roy-Camille et al. [2,3] in the 1960s, has become one of the most widely used fixation techniques in spinal surgery. The technique makes use of the pedicle, which is in terms of biomechanics, the hardest part of the vertebra, as an anchor for the fixation device, enabling improved correction and maintenance of spinaldeformities $[1,4]$. However, narrow pedicles in scoliotic patients pose a risk of injury to the spinal cord, nerve root, and aorta caused by the screw perforating the pedicle $[5,6,7,8,9]$.

The majority of studies on pedicle morphology in patients with adolescent idiopathic scoliosis (AIS) have used two-dimensional (2D) analysis by magnetic resonance imaging (MRI) or computed tomography (CT) $[6,10,11,12]$. To our knowledge, the present study is the first to evaluate pedicle morphology in AIS populations by multidimensional 


\section{Materials and Methods}

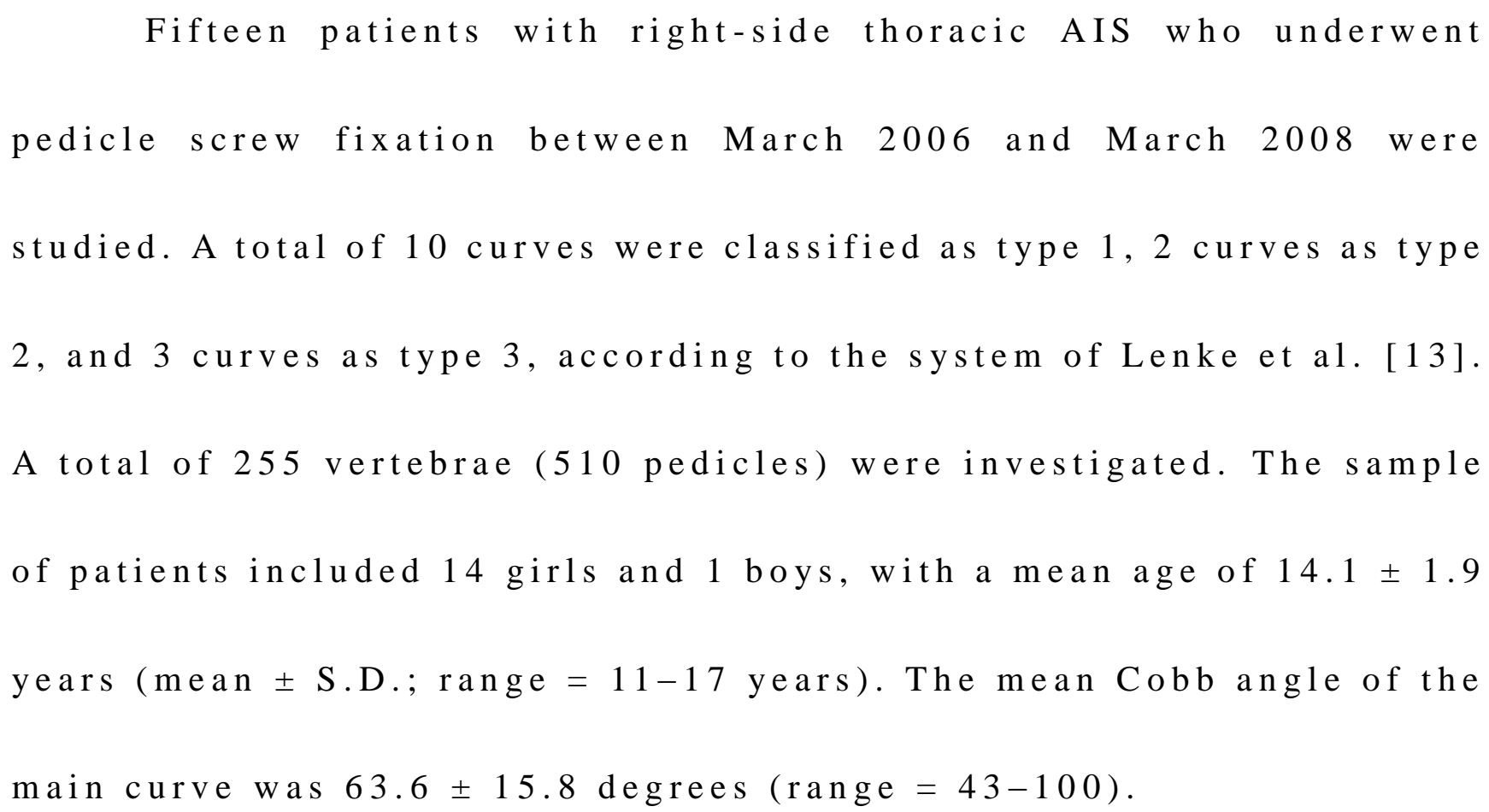


Pedicle Morphology in Idiopathic Scoliosis- 3 -

The CT-based navigation system used was a Stealth Station ${ }^{\circledR}$

TREON ${ }^{\circledR}$ plus (Medtronic, Sofamor Danek, Memphis, TN, USA) surgical navigation system. Pedicle screws were placed in optimal positions using the CT-based navigation system. Lenke's [14] method was used to determine the insertion point of the screw. The pedicle diameter was measured as the widest outer cortical diameter at the narrowest part of the pedicle between T1 and L5. The maximum pedicle screw trajectory length was measured as the distance between the posterior cortical entry point of the pedicle screw and the anterior vertebral cortex in line with the axis of the pedicle. From the various techniques available for pedicle screw placement, we chose the straightforward technique because of its prevalence and its biomechanical superiority over the anatomic technique [15] (Figure 1). The values obtained for the parameters measured using the CT-based navigation system and standard axial CT were compared to evaluate the validity of multidimensional analysis by a CT-based navigation system. Preoperative standard axial CT with a slice thickness of $1.25 \mathrm{~mm}$ was performed in the axial section through the 
entire spine. All CT files were analyzed using a DICOM viewer program

(ExaView LITE; Ziosoft, Tokyo, Japan). All parameters were measured

by the first author (S.K.), who was a neutral party. Window level and

diameter were optimized for the measurement of bony structure [16].

Two parameters of diameter, length on both sides of the pedicle were

measured for each vertebra. Each section of the image was not always

parallel to each intervertebral space. We chose the optimal slice

wherein an insertion point and direction were determined to get the

largest diameter of a screw in every vertebra. We measured the diameter

in the transverse plane as the length from the insertion point to the tip

of the simulated screw (Figure 2).

We selected the screws based on data obtained using the

C T-based navigation system and postoperatively evaluated whether the

screws used were suitable for the pedicles. To evaluate the length of the

inserted screws, we measured the length of the screws placed and

determined the exact length that would have been needed to reach the

far cortex in the postoperative CT scan and compared those data.

To evaluate the diameter of the inserted screws, we measured 
screw positions by postoperative CT._The screw position was evaluated

1 week after the operation using an axial CT scan at each screw axis.

Following Rao et al. [17], the evaluation of screw malposition was

classified as Grade 0 (no apparent violation of the pedicle), Grade $1(<2$

m m perforation of the pedicle, with 1 screw thread outside of the

pedicle), Grade 2 (between 2 and $4 \mathrm{~mm}$ perforation of the pedicle, with

half of the diameter of the screw outside of the pedicle), or Grade $3(>4$

mm or complete perforation of the pedicle), with Grades 2 and 3

representing "violation." On the basis of this grading system, screw

misplacement rates were determined. Medial or lateral perforation of

the pedicle wall was also evaluated.

Paired $t$ - tests were performed using Stat View ( $S$ A S

Institute, Cary, N C, U S A ). M e a n s and standard deviations werecalculated, and the statistical significance was set a t $\mathrm{p}<0.05$. 
Pedicle Morphology in Idiopathic Scoliosis- 6 -

\section{Results}

\section{Mean pedicle diameter}

Mean pedicle diameter is summarized in Table 1. On the left side, the diameters decreased successively from T1, with the smallest value at $\mathrm{T} 7(4.17 \pm 0.78 \mathrm{~mm})$, then increased from T8 to T 12 . Diameters also decreased from L1 to L2, then increased from L3, reaching the maximum at L5 $(9.61 \pm 1.33 \mathrm{~mm})$. On the right side, pedicle diameter decreased from T1, with the smallest value at T4 $(3.73 \pm 0.41 \mathrm{~mm})$, then increased from T5 to T12. Diameters decreased at L1 and increased again from L2, reaching the maximum at L5 $(9.50 \pm 1.50 \mathrm{~mm})$. A mean pedicle diameter less than $4.5 \mathrm{~mm}$ was observed at T3, T4, and T5 on the right side, and at T6, T7, T8, and T9 on the left side. The smallest thoracic pedicle screw diameter that can be used in Japan is usually 4.5 mm. Significant differences were observed on the opposite side at T3, T4, and T5 on the right side, and at T 8 and T9 on the left side $(\mathrm{p}<0.05$; Table 1). On the right side, the mean pedicle diameters of T3 and T4 candidates for upper-instrumented vertebrae were less than $4.0 \mathrm{~mm}$, and 
thus, screw fixation was difficult. In summary, the pedicles on the concave side of the main thoracic curve apex and the proximal thoracic curve apex had significantly narrow diameters $(\mathrm{p}<0.05)$.

The mean pedicle diameter as measured by the CT navigation system and standard axial CT is summarized in Figure 3. The mean pedicle diameter measured by the CT navigation system was larger than that measured by standard axial CT, except for both sides of T 11 , T 12 , and L5 and the left side of L3 and L4. Significant differences were observed as follows: right side, T3 $(\mathrm{p}=0.0009), \mathrm{T} 4(\mathrm{p}=0.000054)$, T5 $(\mathrm{p}=0.0013)$, and T7 $(\mathrm{p}=0.039) ;$ left side, T4 $(\mathrm{p}=0.033)$, T5 $(\mathrm{p}=$ $0.03)$.

\section{Mean maximum pedicle screw trajectory length}

The mean lengths of the longest pedicle screw trajectories are summarized in Table 2. The mean screw lengths were longer on the right side, except for T 8 and T9, but the difference was not statistically significant. The screws gradually became longer from the cranial thoracic spine to the caudal lumbar spine. Screw size 
Pedicle Morphology in Idiopathic Scoliosis- 8 -

feasibility was as follows: screws between $25 \mathrm{~mm}$ and $30 \mathrm{~mm}$ were

feasible from $\mathrm{T} 1 \mathrm{to} \mathrm{T} 5$, screws between $30 \mathrm{~mm}$ and $35 \mathrm{~mm}$ were feasible from T6 to T12, and screws between $35 \mathrm{~mm}$ and $40 \mathrm{~mm}$ were feasible from L1 to L5.

On the left side, all pedicle screw trajectory lengths measured by the CT navigation system were significantly shorter than those measured by standard axial CT: T1 $(p=0.00024)$, T3 $(p=0.010)$, T4 $(p$ $=0.0043), \mathrm{T} 6(\mathrm{p}=0.010), \mathrm{L} 1(\mathrm{p}=0.0010), \mathrm{L} 2(\mathrm{p}=0.0009), \mathrm{L} 3(\mathrm{p}=$ 0.0051). As for the right side in thoracic vertebrae, the pedicle screw lengths measured by the CT navigation system, except for T3, were longer than those measured by standard axial CT, and a significant difference was observed for T7 ( $p=0.019)$; in lumbar vertebrae, the pedicle screw lengths measured by the CT navigation system were shorter than those measured by standard axial CT, and a significant difference was observed for L2 ( $p=0.025$; Figure 4).

\section{Postoperative assessment of screw placement}


The appropriateness of the length of the screw used for surgery

was evaluated. The mean difference between the length of the pedicle measured by the CT navigation system and the length of the screw used was $2.3 \pm 5.0 \mathrm{~mm}$. This implies that the distance from the tip of the screw to the bone cortex in the direction of screw insertion should be $2.3 \mathrm{~mm}$ when the screw is correctly and fully inserted. However, postoperative measurement of the mean distance from the tip of the screw to the bone cortex in the direction of screw insertion by CT resulted in a value of $6.7 \pm 4.3 \mathrm{~mm}$. The difference (about $4.4 \pm 6.0 \mathrm{~mm}$ ) between the theoretical value $(2.3 \mathrm{~mm})$ and the actual value $(6.7 \mathrm{~mm})$ resulted from insufficient insertion of the screw. The values calculated by subtracting the length of the screw used from the pedicle length measured by the CT navigation system on the right and left sides were $3.2 \pm 4.7 \mathrm{~mm}$ and $1.4 \pm 5.1 \mathrm{~mm}$, respectively, showing a significant difference $(p=0.0076)$. These results show that on the left side, the length of the screw selected based on data from the CT navigation system was closer to the pedicle length measured by the same system. However, the distance between the screw tip and the cortical bone as 
measured by postoperative CT showed no significant difference between the right and left sides. The distance of insufficient screw insertion (actually inserted length of screw - [pedicle length as measured by the navigation - distance from the screw tip to the cortical bone of anterior vertebrae]) was $3.8 \pm 5.9 \mathrm{~mm}$ and $5.2 \pm 5.8 \mathrm{~mm}$, respectively, on the right and left sides; thus, the left side showed a statistically greater tendency for insufficient screw insertion $(\mathrm{p}=$ 0.044). It is possible that this insufficient insertion on the left side resulted from the fear of arterial injury. A total of 7 of the 215 screws ( $3.3 \%$ ) perforated the anterior cortex on the right T1, T5, T12, and L1 and on the left T1, T12, and L 1 .

The perforation rate was measured to evaluate the appropriateness of the used screw diameter. Of the 215 pedicle screws, $164(76.3 \%)$ were categorized as Grade $0,47(21.9 \%)$ as Grade 1,3 $(1.4 \%)$ as Grade 2, and $1(0.5 \%)$ as Grade 3 (Figure 5). All medial perforations of Grade 2 or more were on the right side (T6, T7, and T9). Only 1 Grade 3 screw was seen in a right T9 pedicle. Pedicle violation was observed in $1.9 \%(4 / 215)$ of the inserted screws. Lateral screw 
perforation was as follows: right side, T3, T4, T5, T12, L1, and L3, and left side, T11 and T12. No intraoperative complications such as neurovascular injury or adverse clinical consequences occurred because of pedicle perforation.

\section{Discussion}

The study of pedicle morphology for screw placement in scoliosis is important because screw misplacement results in lower pullout strength and could cause serious complications including nerve, blood vessel, or visceral injury $[5,6,7,8,9]$. Multiple reports of using pedicle measurement to identify the morphology of AIS $[10,11,12,18,19]$ have been made, but these used 2D analysis by CT or MRI. Parent et al. [12] measured and compared normal and scoliotic pedicle morphology by fixing each vertebral body to a frame and reconstructing the $3 \mathrm{D}$ image by plotting the vertebrae using a $3 \mathrm{D}$ digitizer. Their results showed significantly narrower scoliotic pedicles on the concave side of the 
main (T8) and proximal (T4) thoracic curves compared to the normal vertebrae. They suggested that pedicle screw insertion for scoliotic patients in the apex of the curve on the concave side should be avoided. Similarly, Liljenqvist et al. [10] measured scoliotic vertebrae by MRI and reported narrower pedicles on the concave side in the apex region of the main thoracic curve (T7-T10) than the convex side.

Takeshita et al. [20] reported on the diameter, length, and direction of pedicle screws by multiplanar reconstruction of CT for various conditions of Japanese scoliotic spine. To our knowledge, the present study is the first to use multidimensional analysis based on a CT-based navigation system to evaluate pedicle morphology only in AIS patients. The results of this study show that the narrowest mean pedicle diameters were observed at T7 and T4 on the left side and right side, respectively. In the study by Takeshita et al. [20]62\% of the concave T3-T9 screw diameters were less than $4 \mathrm{~mm}$, and $37 \%$ of these pedicles did not hold the 4-mm diameter screw even with $25 \%$ expansion. These results indicate that the pedicles on the concave side of the main thoracic curve apex and proximal thoracic curve apex have 
significantly narrow diameters, consistent with previous reports (Table 3). These results highlight the necessity of practicing caution when inserting pedicle screws on the concave side of the main and proximal thoracic curve, and suggest that extrapedicular screws should be considered in the preoperative plan if pedicle screw insertion is judged to be difficult.

Extrapedicular screw placement has been advocated as a safe and effective option to the thoracictranspedicular screw [15,21]. However, screws placed in the extrapedicular position have about $75 \%$ of the pullout failure load of those placed in the transpedicular position [22,23]. These concerns emphasize the value of using a CT navigation system to measure pedicle diameter for transpedicular screw insertion. Although the powerful corrective force and maintenance of segmental pedicle screws is fascinating, surgeons need not always use pedicle screws. They can use other anchoring methods like hooks, wires and polyethylene cables, when preoperative evaluation reveals narrow pedicles that are not appropriate for pedicle screw placement.

Under such conditions, the anatomic trajectory technique could be 
the technique of choice, although the screws placed by the anatomic trajectory are biomechanically weaker than those placed by the straightforward trajectory. However, Takeshita et al.[20] found that pedicles too narrow for the straightforward technique generally had impracticable results for the anatomic technique as well. They did not try to simulate the anatomic trajectory because simulation of screw placement would have been too complicated, and they concluded that future analysis of the feasibility of using the anatomic trajectory is warranted.

Parent et al. [12] reported distinct technical limitations of $2 \mathrm{D}$ analysis by CT or MRI in terms of obtaining sectional images of the narrowest pedicle levels in the vertebrae. They also suggested the necessity of $3 \mathrm{D}$ evaluation of scoliotic change to avoid large measurement error caused by slight changes of image. They measured pedicles by $3 \mathrm{D}$ reconstruction of vertebrae by plotting fixed individual vertebrae using a $3 \mathrm{D}$ digitizer. However, the reconstructed image obtained was irregular.

Liljenqvist et al. [10] stated that CT could control table position 
only in the direction of the sagittal axis, and that table adjustment

along the axis of the coronal plane is not possible. This limitation could cause skewing of vertebral image sections except for in the apex region, resulting in error in measurement.

We compared the diameters and lengths of the pedicles as measured by the CT navigation system and preoperative standard axial CT. The pedicle diameter measured by the CT navigation system was larger than that measured by standard axial CT. Thus, the use of a navigation system could make screw insertion feasible even when evaluation by standard CT judges screw insertion to be impossible. The pedicle length measured by the CT navigation system was shorter on the left side and longer on the right side, compared to that measured by standard CT. This is because of the technical limitations of standard axial CT, and the tilting or rotation of the vertebrae could be the cause. Standard axial CT possibly results in the preoperative selection of a smaller screw diameter, and on the left side, a longer screw length. Because the artery is located on the left side, screw length selection based on standard axial CT carries a higher risk of injury to the artery than 
selection based on the CT navigation system.

Catan et al. [11] measured the morphology of scoliotic pedicle by

MRI, and reported that MRI could not clearly describe the structure of the cortical bone. In our study, the pedicle diameter tended to be wider than in other studies (Table 3 ). The mean pedicle diameter on the concave side of the main thoracic curve has been reported as follows:

$3.8 \mathrm{~mm}$ ( T6) by Parent et al. [12], 2.5 mm (T8) by Liljenqvist et al. [10], $2.6 \mathrm{~mm}$ ( T9) by Thomas et a1. [19], 4.3 mm ( T5, T6, T7) by Catan et al. [11], and 4.0 mm (T3-T9) by Takeshita et al. [20].

The clinical application of $3 \mathrm{D}$ computer-assisted surgery has significantly reduced the rate of screw misplacement $[1,8,22,23,24,25,26]$. Clinical studies have demonstrated an improved accuracy in pedicle screw insertion because of the use of a CT-based navigation system, which eliminates exposure to fluoroscopic radiation. Kotani et al. [5] evaluated the accuracy of pedicle screw placement in posterior scoliosis surgeries with and without the use of computer-assisted surgical techniques. They showed that perforation was observed in $11 \%$ of cases without the use of computer-assisted 
surgical techniques and $1.8 \%$ of cases with the use of such techniques.

We predicted screw diameter and length from the preoperative plan with the CT navigation system, and the rate of Grade 2 or more perforation ( 4 right pedicles; levels T6, T7, and T9) was $1.9 \%$. In our department, pedicle insertion on the left and right sides is performed by a senior surgeon (J.T.) and fellows, respectively, and thus, the occurrence of perforation could be related to the learning curve of the surgeon. The absence of left medial perforation of the thoracic vertebrae indicates the value of the CT navigation system.

In our study, the pedicle diameters were wider than those found in previous studies. This is possibly because the previous studies used 2D analysis, which could not obtain accurate sectional planes, whereas the multidimensional analysis based on CT-based navigation used in the present report enabled screw insertion in favorable directions. Pedicle screws were placed in optimal positions by using the CT-based navigation system. However, the reported pedicle diameters found by Takeshita et al. [20] by using multidimensional analysis based on CT-based navigation were narrower compared to our data, resulting 
from the inclusion of syndrome-related scoliosis cases in the study by Takeshita et al.

Finally, in this study, the feasible screw length tended to be between $25 \mathrm{~mm}$ and $30 \mathrm{~mm}$ from $\mathrm{T} 1 \mathrm{to}$ T 5 , between $30 \mathrm{~mm}$ and $35 \mathrm{~mm}$ from T6 to T12, and between $35 \mathrm{~mm}$ and $40 \mathrm{~mm}$ from L1 to L5. These results could be used as an indicator for screw length selection. Takeshita et al. [20] reported that $11 \%$ of the convex T4-T8 diameters were $25 \mathrm{~mm}$ or less, and required careful screw insertion.

In conclusion, the pedicle diameter in patients with AIS is narrower on the concave side of the scoliotic curve, and therefore, caution should be exercised when inserting screws on this side. A wider angle and trajectory selection of screw insertion achieved by multidimensional analysis with a CT-based navigation system resulted in wider pedicle diameter values, compared to the previous studies that used 2 D analysis systems. 


\section{References}

1) Boos N, Webb JK. Pedicle screw fixation in spinal disorders: a European view. Eur Spine J. 1997;6:2-18.

2) Roy-Camille R, Saillant G, Mazel C. Internal fixation of the lumbar spine with pedicle screw plating. Clin Orthop. 1986;203:7-17.

3) Roy-Camille R, Saillant G, Mazel C. Plating of thoracic, thoracolumbar, and lumbar injuries with pedicle screw plates. Orthop Clin North Am. 1986;17:147-59.

4) Suk SI, Lee CK, Kim WJ, et al. Segmental pedicle screw fixation in the treatment of thoracic idiopathic scoliosis. Spine. $1995 ; 20: 1399-405$.

5) Kotani Y, Abumi K, Ito M, et al. Accuracy analysis of pedicle screw placement in posterior scoliosis surgery. Spine. 2007;32:1543-50.

6) Lonstein JE, Denis F, Perra JH, et al. Complication associated with pedicle screws. J Bone Joint Surg Am. 1991;81:1519-28.

7) Minor ME, Morrissey NJ, Peress R, et al. Endovascular treatment of an iatrogenic thoracic aortic injury after spinal instrumentation: 
case report. J Vasc Surg. 2004.;39:893-6.

8) Papin P, Arlet V, Marchesi D, et al. Unusual presentation of spinal cord compression related to misplacement pedicle screws in thoracic scoliosis. Eur Spine J. 1999;8:156-60.

9) West JL, Ogilvie JW, Bradford DS. Complications of the variable screw plate pedicle screw fixation. Spine. 1991;16:576-9.

10) Liljenqvist UR, Allkemper T, Hackenberg L, et al. Analysis of vertebral morphology in idiopathic scoliosis with use of magnetic resonance imaging and multiplanar reconstruction. J Bone Joint SurgAm. 2002;84-A(3):359-68.

11) Catan H, Buluc L, Anik Y, et al. Pedicle morphology of the thoracic spine in preadolescent idiopathic scoliosis: magnetic resonance supported analysis. Eur Spine J. 2007;16(8):1203-8.

12) Parent S, Labelle H, Skalli W, et al. Thoracic pedicle morphometry in vertebrae from scoliotic spines. Spine. 2004;29:239-48.

13 ) Lenke LG, Betz RR, Harms J, et al. Adolescent idiopathic scoliosis: a new classification to determine extent of spinal arthrodesis. $J$ Bone Joint Surg. 2001;83:1169-81. 
14) Lenke LG. Debate: Resolved, a 55[degrees]? right thoracic adolescent idiopathic scoliotic curve should be treated by posterior spinal fusion and segmental instrumentation using thoracic pedicle screws. J Pediatr Orthop. 2004;24:329-34.

15) White KK, Oka R, Mahar AT, et al. Pullout strength of thoracic pedicle screw instrumentation. Comparison of the transpedicular and extrapedicular techniques. Spine. 2006;31:E355-8.

16) Lehman RA Jr., Polly DW Jr., Kuklo TR, et al. Straight-forward versus anatomic trajectory technique of thoracic pedicle screw fixation: a biomechanical analysis. Spine 2003;28:2058-65.

17) Rao G, Brofke DS, Rondina M, et al. Inter-and intraobserver reliability of computed tomography in assessment of thoracic pedicle screw placement. Spine 2003;28:2527-30.

18) Takahashi J, Hirabayshi H, Hashyidate H, et al. Accuracy of multi-level registration in image-guided pedicle screw insertion for adolescent idiopathic scoliosis. Spine. 2010;35(3):347-52.

19) Thomas J. Gilbert J, Robert B, et al. Pedicule anatomy in a parent with severe early-onset scoliosis: Can pedicle screws be safely 
inserted? J Spinal Tech. 2005;18:360-363.

20 ) Takeshita K, Maruyama T, Chikuda H, et al. Diameter, length, and direction of pedicle screws for scoliotic spine. Spine. 2009;34: $798-803$.

21 ) Suk S-I, Kim W-J, Lee SM, et al. Thoracic pedicle screw fixation in spinal deformities: are they really safe? Spine. 2001;26:2049-57.

22) Morgenstern W, Ferguson SJ, Berey S, et al. Posterior thoracic extrapedicular fixation: a biomechanical study. Spine. $2003 ; 28: 1829-35$.

23 ) Schwarzenbach O, Berlemann U, Jost B, et al. Accuracy of computer-assisted pedicle screw placement: an in vivo computed tomography analysis. Spine. 1997;22:452-8.

24 ) Laine T, Lund T, Ylikoski M, et al. Accuracy of pedicle screw insertion with and without computer assistance. A randomized controlled clinical study in 100 consecutive patients. Eur Spine J. $2000 ; 9: 235-40$

25 ) Schlenzka D, Laine T, Lund T. Computer-assisted spine surgery. Eur Spine J. 2000;9(supp1):S 57-64. 
Pedicle Morphology in Idiopathic Scoliosis- 23 -

26) Ebmeler K, Giest K, Kalff R. Intraoperative computerized

tomography for improved accuracy of spinal navigation in pedicle screw placement of the thoracic spine. Acta Neurochir Suppl. $2003 ; 85: 105-13$. 
Pedicle Morphology in Idiopathic Scoliosis- 24 -

\section{Figure Legends}

Figure 1. Screen image of Stealth Station ${ }^{\circledR}$ TREON ${ }^{\circledR}$ plus during measurement

Figure 2. Illustration of a thoracic vertebra showing the pedicle length (AC) and the transverse pedicle diameter (DE)

Figure 3. Comparison of mean pedicle diameter as measured by the computed tomography (CT) navigation system and standard axial CT Diameters measured by the CT navigation system were larger than those measured by standard axial CT, except for both T11, T12, and L5 and left L3 and L4. p < $0.05^{*}, \mathrm{p}<0.01 * *, \mathrm{p}<0.001 * * *$.

Figure 4. Comparison of mean pedicle trajectory length as measured by the computed tomography (CT) navigation system and standard axial C T 
Pedicle Morphology in Idiopathic Scoliosis- 25 -

On the left side, all pedicle lengths measured by the CT navigation system were shorter than those measured by standard CT. On the right side, the pedicle lengths measured by the CT navigation system in the thoracic and lumbar levels were longer and shorter, respectively, than those measured by standard axial CT. p < $0.05^{*}, \mathrm{p}<0.01^{* *}, \mathrm{p}<$ $0.001 * * *$.

Figure 5. Actual screw position of each vertebral level 
Table 1. Mean pedicle diameter and comparison between left side and right side

\begin{tabular}{|c|c|c|c|}
\hline Level & $\begin{array}{c}\text { Left } \\
(\mathrm{mm})\end{array}$ & $\begin{array}{c}\text { Right } \\
(\mathrm{mm})\end{array}$ & p value \\
\hline T1 & $5.81 \pm 0.89$ & $5.79 \pm 1.06$ & n.s. \\
\hline T2 & $5.15 \pm 0.74$ & $5.02 \pm 0.84$ & n.s. \\
\hline T3 & $4.85 \pm 0.44$ & $3.87 \pm 0.59$ & $\mathrm{p}<0.01$ \\
\hline T4 & $4.65 \pm 0.44$ & $3.73 \pm 0.41$ & $\mathrm{p}<0.01$ \\
\hline T5 & $4.79 \pm 0.59$ & $4.09 \pm 0.76$ & $\mathrm{p}<0.01$ \\
\hline T6 & $4.45 \pm 0.59$ & $4.62 \pm 0.89$ & n.s. \\
\hline T7 & $4.17 \pm 0.78$ & $4.77 \pm 0.75$ & n.s. \\
\hline T8 & $4.28 \pm 0.73$ & $5.07 \pm 0.86$ & p<0.05 \\
\hline T9 & $4.29 \pm 0.81$ & $5.24 \pm 0.68$ & p<0.01 \\
\hline T10 & $4.96 \pm 1.05$ & $5.26 \pm 0.84$ & n.s. \\
\hline T11 & $6.13 \pm 1.45$ & $6.27 \pm 1.19$ & n.s. \\
\hline T12 & $6.34 \pm 1.22$ & $6.45 \pm 1.62$ & n.s. \\
\hline L1 & $5.87 \pm 1.22$ & $5.79 \pm 1.10$ & n.s. \\
\hline L2 & $5.65 \pm 0.94$ & $6.16 \pm 0.90$ & n.s. \\
\hline L3 & $7.05 \pm 1.21$ & $7.38 \pm 1.16$ & n.s. \\
\hline L4 & $8.37 \pm 1.18$ & $8.42 \pm 1.23$ & n.s. \\
\hline L5 & $9.61 \pm 1.33$ & $9.50 \pm 1.50$ & n.s. \\
\hline Tean & & & \\
\hline
\end{tabular}

: Mean pedicle diameter was less than $4.5 \mathrm{~mm}$ 
Table 2. Mean maximum pedicle screw trajectory length

\begin{tabular}{|c|c|c|c|}
\hline Level & $\begin{array}{l}\text { Left } \\
(\mathrm{mm})\end{array}$ & $\begin{array}{l}\text { Right } \\
(\mathrm{mm})\end{array}$ & $\mathrm{p}$ value \\
\hline $\mathrm{T} 1$ & $24.98 \pm 3.73$ & $25.71 \pm 3.44$ & n.s. \\
\hline $\mathrm{T} 2$ & $28.55 \pm 3.21$ & $31.45 \pm 3.46$ & n.s. \\
\hline T3 & $28.35 \pm 2.46$ & $31.89 \pm 3.18$ & n.s. \\
\hline $\mathrm{T} 4$ & $28.26 \pm 2.60$ & $33.93 \pm 3.22$ & n.s. \\
\hline $\mathrm{T} 5$ & $29.31 \pm 3.32$ & $34.94 \pm 4.53$ & n.s. \\
\hline T6 & $30.23 \pm 5.06$ & $35.23 \pm 5.06$ & n.s. \\
\hline $\mathrm{T} 7$ & $30.99 \pm 4.47$ & $34.67 \pm 3.89$ & n.s. \\
\hline $\mathrm{T} 8$ & $34.23 \pm 4.86$ & $33.03 \pm 5.34$ & n.s. \\
\hline T9 & $35.33 \pm 4.05$ & $34.79 \pm 4.15$ & n.s. \\
\hline T10 & $34.47 \pm 4.67$ & $34.89 \pm 4.03$ & n.s. \\
\hline $\mathrm{T} 11$ & $31.30 \pm 4.64$ & $34.15 \pm 4.82$ & n.s. \\
\hline $\mathrm{T} 12$ & $33.93 \pm 7.45$ & $35.45 \pm 4.80$ & n.s. \\
\hline L1 & $36.63 \pm 5.31$ & $40.35 \pm 6.95$ & n.s. \\
\hline $\mathrm{L} 2$ & $39.06 \pm 4.08$ & $42.10 \pm 3.60$ & n.s. \\
\hline L3 & $40.05 \pm 4.24$ & $43.22 \pm 4.49$ & n.s. \\
\hline $\mathrm{L} 4$ & $41.17 \pm 5.00$ & $42.09 \pm 3.07$ & n.s. \\
\hline L5 & $39.70 \pm 5.09$ & $41.16 \pm 4.30$ & n.s. \\
\hline
\end{tabular}


Table 3. The reports of the mean thoracic pedicle diameter (mm)

\begin{tabular}{|c|c|c|c|c|c|c|c|c|c|c|c|c|}
\hline & \multicolumn{2}{|c|}{ Parent et al. } & \multicolumn{2}{|c|}{ Liljenqvist et al. } & \multicolumn{2}{|c|}{ Thomas et al. } & \multicolumn{2}{|c|}{ Catan et al. } & \multicolumn{2}{|c|}{ Takeshita et al. } & \multicolumn{2}{|c|}{ Our study } \\
\hline & Left & Right & Left & Right & Left & Right & Left & Right & Left & Right & Left & Right \\
\hline $\mathrm{T} 1$ & 6.7 & 6.8 & & & & & 5.1 & 4.8 & 6.0 & 6.3 & 5.8 & 5.8 \\
\hline $\mathrm{T} 2$ & 5.0 & 4.7 & & & & & 4.7 & 4.9 & 4.9 & 5.5 & 5.2 & 5.0 \\
\hline $\mathrm{T} 3$ & 4.3 & 3.2 & & & & & 4.4 & 4.4 & 3.3 & 4.7 & 4.9 & 3.9 \\
\hline $\mathrm{T} 4$ & 4.0 & 2.6 & & & & & 4.5 & 4.5 & 2.7 & 4.3 & 4.7 & 3.7 \\
\hline $\mathrm{T} 5$ & 4.0 & 3.6 & 3.1 & 3.7 & 4.6 & 3.5 & 4.3 & 4.5 & 3.0 & 4.5 & 4.8 & 4.1 \\
\hline $\mathrm{T} 6$ & 3.8 & 4.7 & & & 4.5 & 4.8 & 4.3 & 4.6 & 3.5 & 4.4 & 4.5 & 4.6 \\
\hline $\mathrm{T} 7$ & 4.0 & 5.2 & 3.1 & 4.1 & 3.7 & 5.7 & 4.3 & 4.7 & 3.7 & 4.6 & 4.2 & 4.8 \\
\hline $\mathrm{T} 8$ & 4.0 & 5.2 & 2.5 & 4.2 & 3.4 & 6.4 & 4.4 & 4.7 & 3.8 & 4.6 & 4.3 & 5.1 \\
\hline $\mathrm{T} 9$ & 4.9 & 5.4 & 3.3 & 4.2 & 2.6 & 6.1 & 5.1 & 4.8 & 4.1 & 4.9 & 4.3 & 5.2 \\
\hline $\mathrm{T} 10$ & 5.8 & 6.1 & 4.2 & 5.0 & 2.6 & 7.8 & 5.0 & 4.9 & 5.2 & 6.0 & 5.0 & 5.3 \\
\hline T11 & 7.2 & 7.6 & 5.4 & 5.7 & 3.4 & 8.0 & 5.2 & 5.7 & 6.7 & 7.2 & 6.1 & 6.3 \\
\hline $\mathrm{T} 12$ & 7.2 & 7.1 & 5.2 & 5.9 & 5.0 & 7.2 & 5.7 & 5.9 & 6.8 & 7.1 & 6.3 & 6.5 \\
\hline
\end{tabular}


Figure (TIF or EPS Only!!! Resolution of at least $300 \mathrm{dpi!)}$
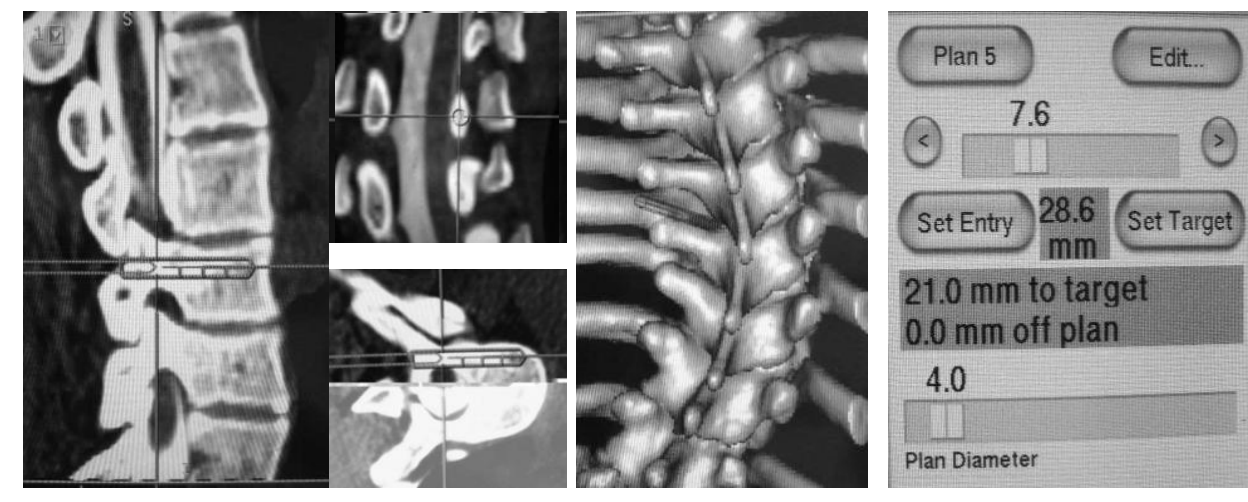

$21.0 \mathrm{~mm}$ to target

$0.0 \mathrm{~mm}$ off plan

4.0

Plan Diameter 


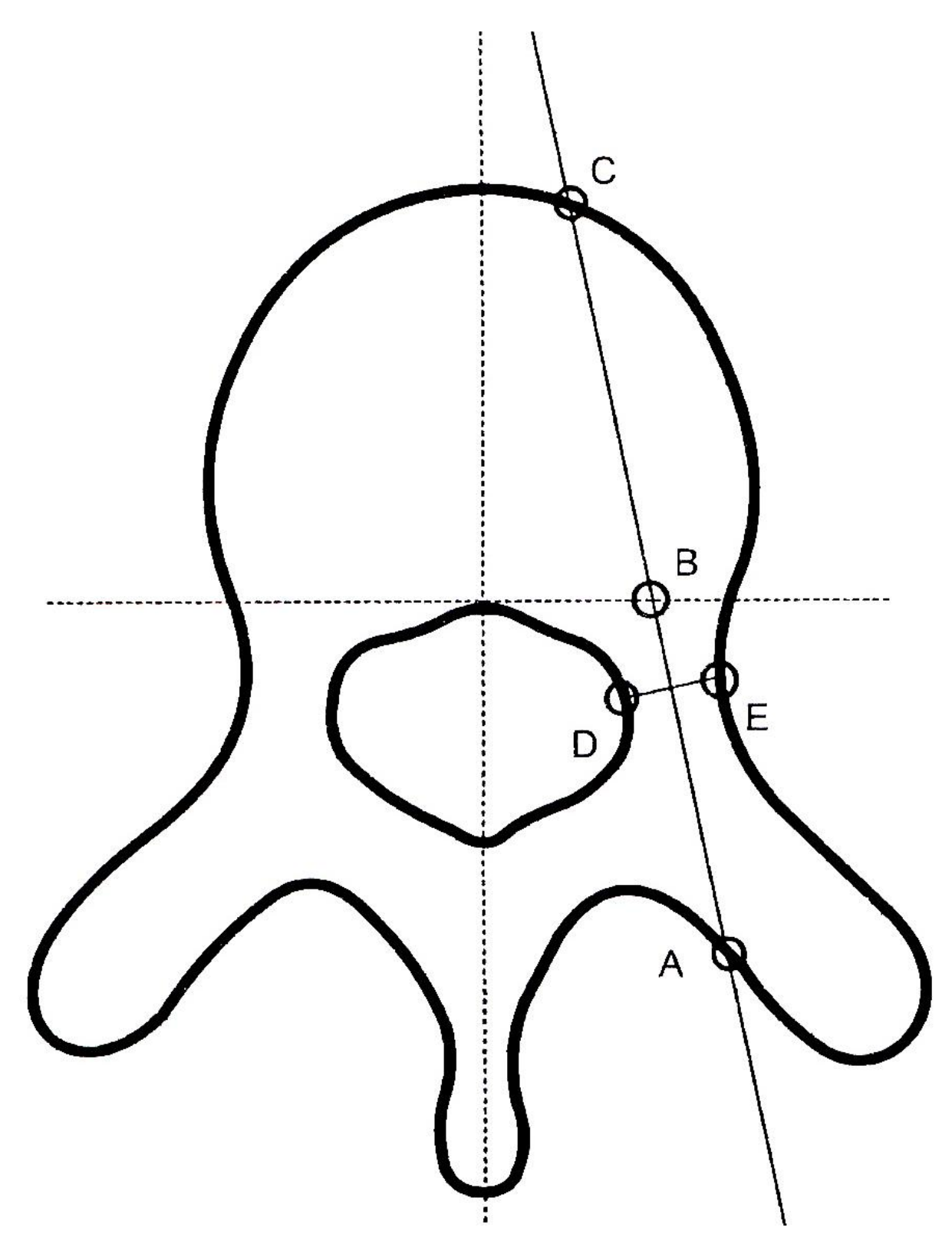



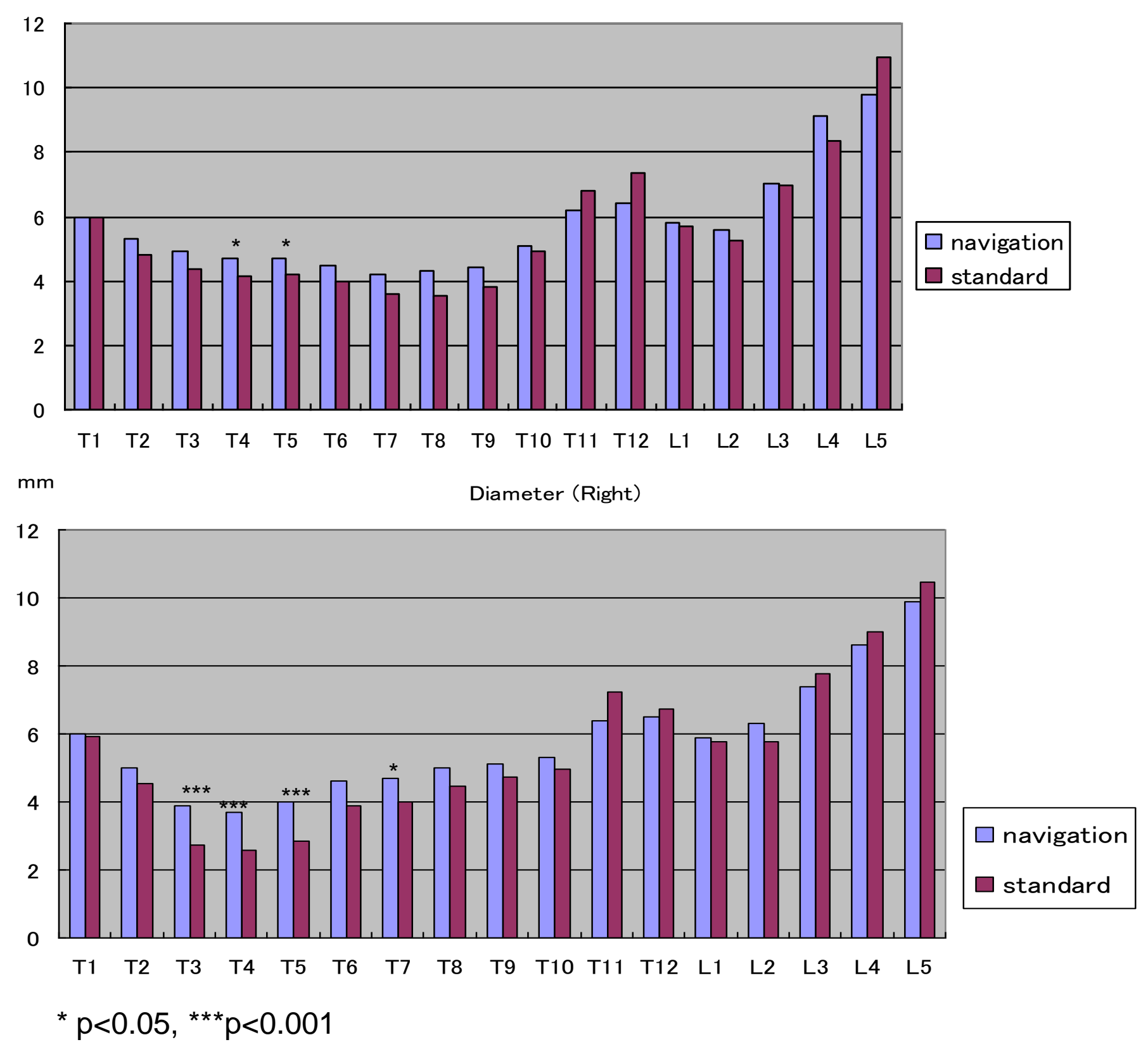

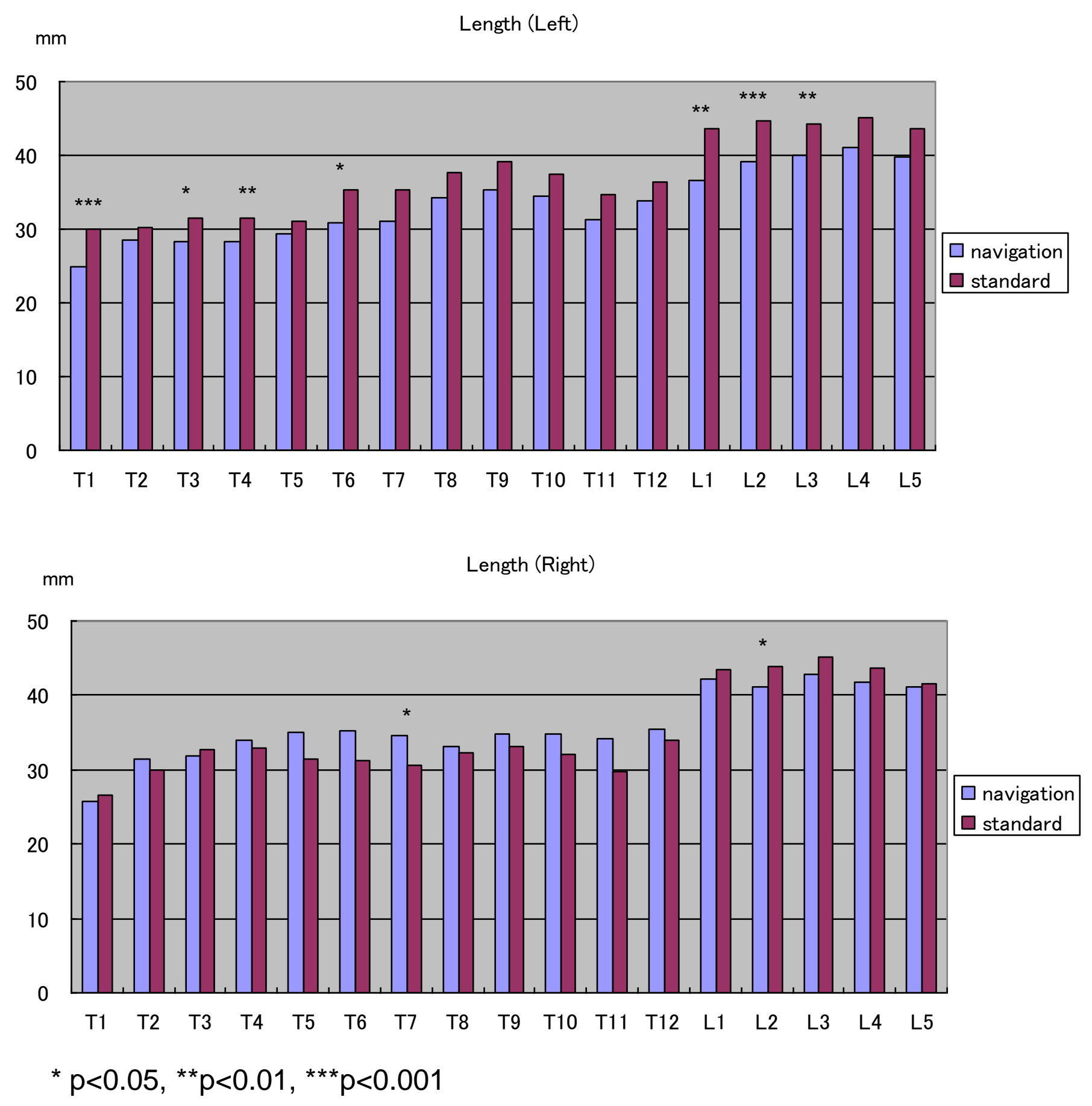
Number of pedicles

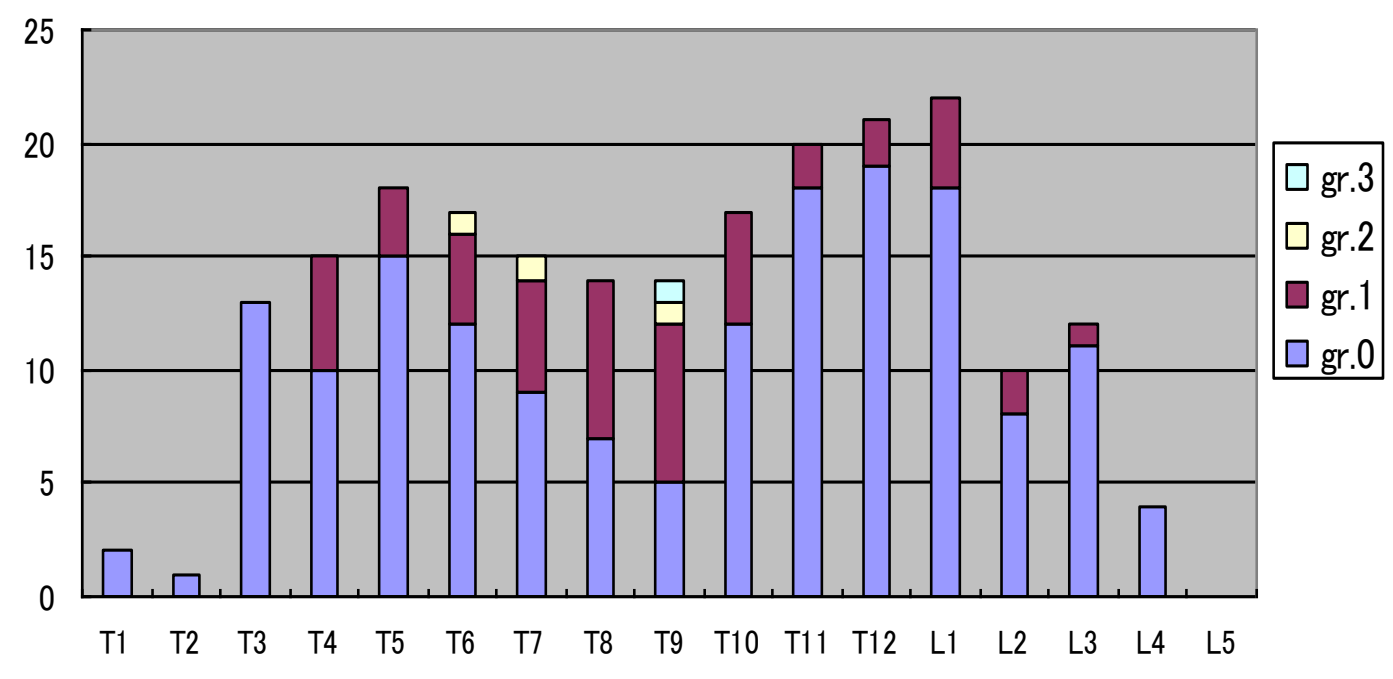

